\title{
External nasal valve collapse: validation of novel outcome measurement tool*
}

\author{
Anne-Lise Poirrier, Suki Ahluwalia, Ivor Kwame, Ha Chau, \\ Melissa Bentley, Peter Andrews
}

The Royal National Throat Nose and Ear Hospital, 330 Grays Inn Road, London, WC1X 8DA, United Kingdom

\author{
Rhinology 52: 127-132, 2014
}

DOl:10.4193/Rhino13.051

*Received for publication:

May 6, 2013

Accepted: October 8, 2013

\section{Summary}

Background: We aim to validate a clinical scoring system of external nasal valve collapse. External nasal valve collapse is a rare and challenging condition. We attempted to simplify the examination of the external valve, the surgical planning and the outcome measure. To validate our external valve score, we first assessed its reliability (inter-rater agreement and test-retest repeatability). We secondly considered the clinical relevance by using our scoring system in patients undergoing septorhinoplasty for external valve collapse.

Methodology: For validation, 16 Rhinologists scored patients separately on two occasions. For the clinical relevance, 26 patients with external valve collapse were scored pre- and post-operatively (responsiveness). The external valve score was correlated to peak nasal inspiratory flow.

Results: The devised scoring system was reliable (substantial agreement between 16 surgeons with reproducibility over time). All patients in our prospective series showed significant improvement in their external valve score. The quality of life measured by the SNOT-22 tool showed significant improvement after surgery.

Conclusion: External nasal valve collapse can be diagnosed and graded using this simple scoring system in the outpatient clinic. This paper reinforces the pivotal role of septorhinoplasty surgery in nasal airway reconstruction and the ongoing need to quantify success.

Key words: outcome, functional septorhinoplasty, nasal obstruction, external nasal valve, lateral wall insufficiency

\section{Introduction}

The anatomical areas of nasal obstruction can be broadly divided into septum, turbinates and the nasal valve area. The nasal valve area can be divided into external and internal nasal valves ${ }^{(1,2)}$. The external nasal valve comprises the alar cartilages, the nasal wing and the columella. The internal nasal valve is the angle formed by the junction of the upper lateral cartilages with the nasal septum. External Nasal Valve Collapse (ENVC) refers to a lateral nasal wall insufficiency and subsequent alar collapse, which is visible on quiet and deep inspiration. Up to now, a quick and reproducible clinical scoring system for ENVC has not been universally adopted. ENVC is more commonly dynamic but can be static in congenital or traumatic conditions.

ENVC occurs when there is a disruption of the normal external nasal valve architecture with typically a weakness in the intermediate and lateral crural complex. This weakness results in a dynamic medialisation of this complex based on the Bernoulli's principle whereby airflow entering a narrow segment accelerates and drops intraluminal pressure ${ }^{(3,4)}$. In certain cases, increased nasal resistance (e.g. due to anterior septal deviation or turbinate hypertrophy) may mimic a controlateral ENVC with the sagging of the alar rim during deep inspiration. In these cases, correction of the primary obstacle (e.g. by septoplasty and/or turbinoplasty) is entirely suitable to treat the nasal obstruction ${ }^{(5)}$. 
Pure ENVC is unusual and accounts for $10 \%$ of the lead author's nasal airway reconstruction practice, but at the same time makes up a significant number of tertiary referrals. The surgical correction is often challenging ${ }^{(6)}$. The process requires the ability to grade severity of collapse, to determine method of reconstruction and finally to quantify success and efficacy of treatment ${ }^{(6,7)}$. A clinical grading system for severity of ENVC both at rest and on dynamic inspiration has not been evaluated although breath rite grading systems looking at lower lateral cartilage collapse have been introduced by Gruber and colleagues ${ }^{(8)}$.

A myriad of techniques have been described to rectify nasal obstruction caused by external nasal valve collapse ${ }^{(1,9,10)}$. The number of surgical techniques often reflects uncertainty in choice of technique ${ }^{(1,11)}$. Nasal airway reconstruction surgeons have the responsibility to provide evidence for their quality of care and effectiveness in surgical treatment. The aim of this study is to validate an external nasal valve collapse outcome measurement tool.

\section{Materials and methods}

\section{ENVC grading system}

We have devised a grading system that measures external nasal valve collapse in each nostril both at rest and on deep inspiration. The external nasal valve is assessed in the clinic enabling both static and dynamic abnormalities to be measured. A score of 0 signifies no collapse, 1 signifies collapse of the external nasal valve but not touching the septocollumella complex and 2 signifies complete collapse with touching of the nasal septum. A maximum score of 4 is given for each nostril at rest and on deep inspiration (Figure 1). This system also allows for assessment of a fixed congenital/scarred collapse, which will be apparent both at rest and potentially exacerbated on deep inspiration. For ease of validation, we used photographs which depict the three levels of ENVC.

\section{Study design}

The study design is summarized in Figure 2.

\section{ENVC grading system reliability}

To evaluate this system, we analyzed inter-rater reliability (score stability when taken by different observers) by kappa of agreement and test-retest reliability (score stability taken by a single observer over time with repeated testing). Sixteen rhinologists assessed photographs of 4 patients with various degrees of ENVC, on gentle and deep inspiration, on two occasions separated by 1 year. The rhinologists were experienced ENT surgeons

A.

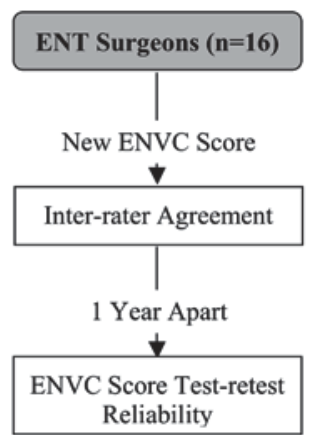

B.

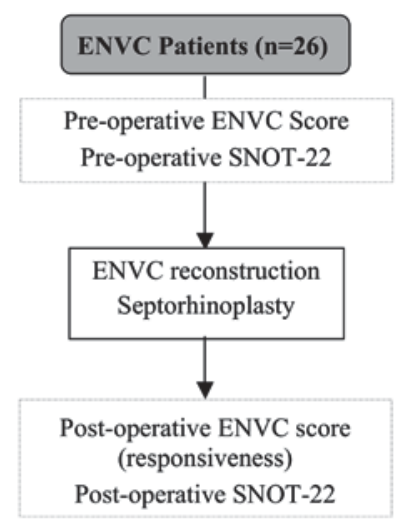

Figure 2. Study Design. A. ENVC grading system reliability. B. Clinical relevance of ENVC grading system. ENVC = External Nasal Valve Collapse. SNOT-22 = Sino-Nasal Outcome Test-22.
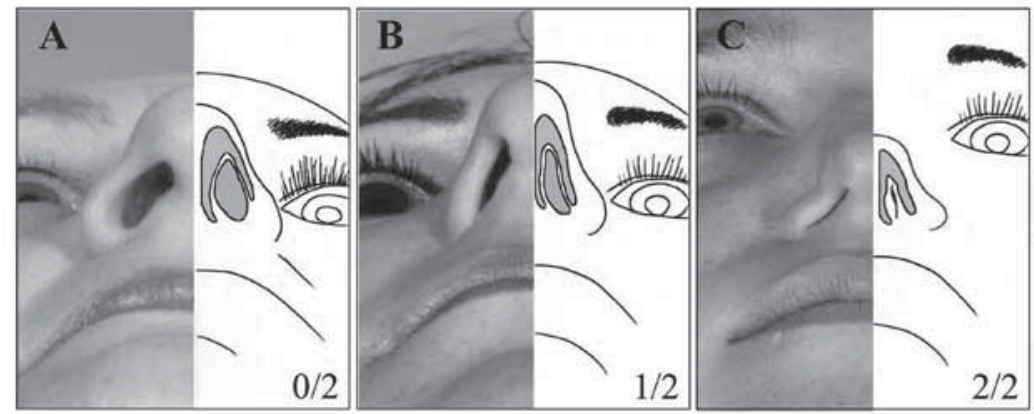

\begin{tabular}{|llc|}
\hline & & Score \\
\hline Right & gentle inspiration & $/ 2$ \\
& deep inspiration & $/ 2$ \\
\hline \multirow{2}{*}{ Left } & Total & 14 \\
\hline & gentle inspiration & $/ 2$ \\
& deep inspiration & 12 \\
\hline & Total & 14 \\
\hline
\end{tabular}

Figure 1. ENVC score. $0=$ no significant movement $(A) ; 1=$ noteworthy movement of the alar volume but not touching the septum/columella $(B) ; 2=$ complete collapse $(C)$. Both sides are scored separately after quiet and deep inspiration and summed with a maximum score of 4 for each side. 


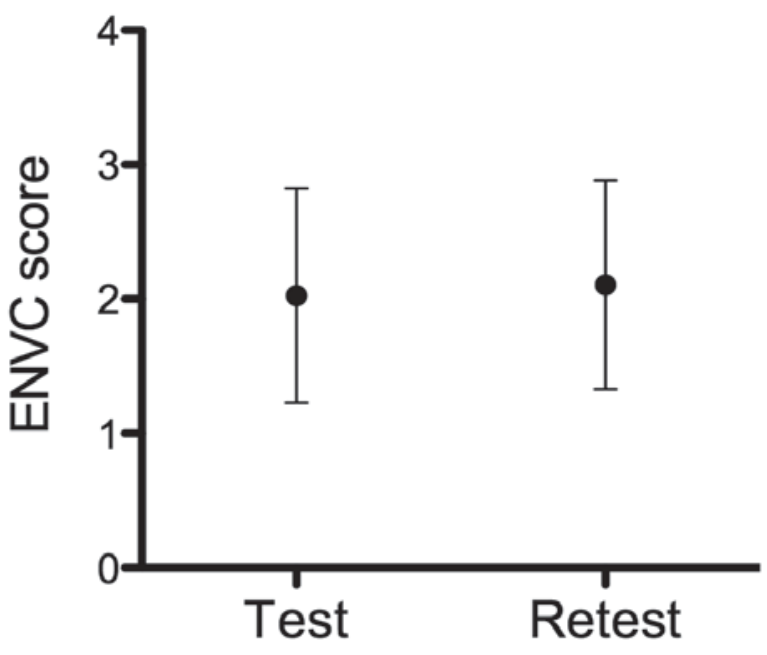

Figure 3. Test-retest reliability measures the stability of an instrument over time with repeated testing (mean \pm SD). Sixteen ENT surgeons scored patients on two different occasions (one year apart) and we examined the correlation between scores. Test-retest reliability coefficient was 0.81 , indicating a good correlation.

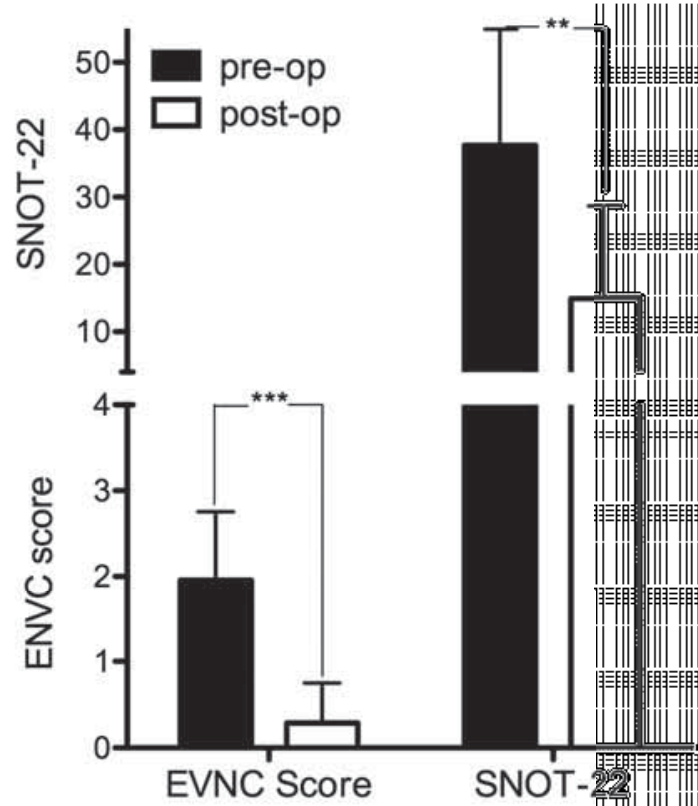

Figure 4. ENVC score (left) and SNOT-22 (right) improved significantly after septorhinoplasty (mean $\pm S D$; respectively $p<0.0001[* * *]$ and $p=$ $0.0010[* *])$. either involved in the rhinology clinics at the Royal National Throat Nose and Ear Hospital or consultant rhinology colleagues from other hospitals. They were blinded to the score attributed by other surgeons, and to the first score at the second evaluation.

\section{Clinical relevance of ENVC grading system}

The ability of our ENVC grading system to reflect expected differences was examined in a separate group of patients. A prospective observational case series was conducted over five years in which 26 patients underwent ENVC nasal airway reconstruction under general anaesthesia. Informed consent was obtained from the study subjects and data were collected in an anonymised database. The ENVC score was measured for each patient preoperatively and at 6 months postoperatively. In all cases, a septal deviation was absent or the obstructing septum had been corrected previously by another surgeon. The turbinates were judged to be of normal size preoperatively and the internal nasal valve was not clinically contributing to nasal blockage. The exclusion criteria included contraindications to surgery, age under 16 , inability to give consent, concomitant functional endoscopic sinus surgery or other nasal airway procedures. We analyzed the responsiveness of our ENVC grading system, i.e. its ability to produce different scores pre- and postoperatively, using the paired Wilcoxon test.

The clinical interpretability of our ENVC score was studied by comparing our score to the validated SNOT-22 questionnaire and to peak nasal inspiratory flow, using the Spearman's corre- lation. Information was also collected for the following: age, sex, clinical history and surgical technique. The influence of factors such as gender or chosen surgical technique was analysed by 2-way ANOVA after adjusting for matching.

\section{Surgical technique}

This article presents the ENVC data preoperatively and 6 months postoperatively. Mild ENVC (score < 2/4) were treated through an endonasal approach using alar batten grafts ${ }^{(12)}$. Moderate and severe ENVC reconstructions (score $>2 / 4$ ) were performed through an external septorhinoplasty approach using either conchal or costal cartilage grafts to reconstruct the intermediate and medial crura. All procedures were conducted by the senior author.

\section{Results}

\section{ENVC grading system reliability}

The agreement between 16 ENT surgeons for ENVC score was substantial (Inter-rater kappa degree of agreement ranged from 0.78 to 1.0 ), showing that this is a reliable score. The left and right ENVC scores attributed to each subject and their respective degree of agreement are shown in Table 1. The test-retest reproducibility was analyzed from the evaluation of the same 16 ENT surgeons one year apart, and resulted in a correlation of 0.81 indicating a high reliability over time (Figure 3 ).

\section{Patient characteristics}

Twenty-six patients underwent ENVC reconstruction. There was 
Table 1. Agreement between 16 ENT surgeons grading 4 different levels of ENVC.

\begin{tabular}{|lcc|}
\hline $\begin{array}{c}\text { Mean ENVC score } \\
(\mathbf{n}=\mathbf{1 6})\end{array}$ & $\begin{array}{c}\text { Inter-rater kappa } \\
\text { degree of agreement }\end{array}$ \\
\hline $\begin{array}{l}\text { Subject 1, Left } \\
\text { Subject 1, Right }\end{array}$ & 2.813 & 0.819 \\
\hline Subject 2, Left & 1.938 & 0.895 \\
\hline Subject 2, Right & 1.969 & 0.906 \\
\hline Subject 3, Left & 3.031 & 0.880 \\
\hline Subject 3, Right & 0.000 & 1.000 \\
\hline Subject 4, Left & 1.031 & 0.906 \\
\hline Subject 4, Right & 1.000 & 1.000 \\
\hline
\end{tabular}

Table 2. Demographic and clinical data recorded in patients treated for external nasal valve collapse.

\begin{tabular}{|c|c|c|}
\hline \multicolumn{3}{|c|}{ Patients' Data } \\
\hline \multirow{3}{*}{$\begin{array}{l}\text { Demographic } \\
\text { Data }\end{array}$} & Total, No & 26 \\
\hline & Age, mean ( $\pm S D), y r$ & $42.4( \pm 14.5)$ \\
\hline & Sex, No (\%) & $\begin{array}{c}13 \mathrm{M}(50 \%) \\
13 \mathrm{~F}(50 \%)\end{array}$ \\
\hline $\begin{array}{l}\text { History \& } \\
\text { Comorbidities }\end{array}$ & $\begin{array}{l}\text { Facial injury, No (\%) } \\
\text { Previous septorhinoplasty, No (\%) } \\
\text { Cleft palate, No (\%) } \\
\text { Allergic rhinitis, No (\%) } \\
\text { Wegener disease, No (\%) } \\
\text { Sarcoidosis, No (\%) }\end{array}$ & $\begin{array}{ll}8 & (30.8 \%) \\
13 & (50 \%) \\
2 & (7.7 \%) \\
1 & (3.8 \%) \\
1 & (3.8 \%) \\
1 & (3.8 \%)\end{array}$ \\
\hline $\begin{array}{l}\text { Surgical } \\
\text { technique }\end{array}$ & $\begin{array}{l}\text { Alar batten graft, No (\%) } \\
\text { LLC repair with conchal graft, No (\%) } \\
\text { LLC repair with rib graft, No (\%) }\end{array}$ & $\begin{array}{l}3(11.5 \%) \\
17(65.4 \%) \\
6(23.1 \%)\end{array}$ \\
\hline
\end{tabular}

no gender preponderance. The mean age was 42.4 years $( \pm 14.5$ years). No correlation between the ENVC score and age was found (Spearman $r=0.018$ ). Thirteen subjects had a previous history of septorhinoplasty, eight had a history of facial injury (including 2 patients who underwent nose mobilization under anaesthesia), two had a history of cleft palate reconstruction, one presented with Wegener's disease and another with sarcoidosis. Demographic and clinical data are summarized in Table 2.

\section{ENVC grading system responsiveness}

All patients reported improved nasal airflow and aesthetics. The mean preoperative ENVC score was $3.62( \pm 0.20)$. The mean postoperative score was $0.52( \pm 0.13)$. The surgical improvement measured by the ENVC score was highly significant $(p<0.0001)$. ENVC scores pre- and postoperatively are shown in Table 3 and Figure 4. The postoperative score improved in all patients
Table 3. ENVC scores, SNOT-22 and peak nasal inspiratory flow measurements pre- and postoperatively.

\begin{tabular}{|c|c|c|}
\hline Measure & Preoperative & Postoperative \\
\hline ENVC score, mean $( \pm S D) / 4$ & $1.96( \pm 0.79)$ & $0.29( \pm 0.45)$ \\
\hline SNOT-22, mean $( \pm$ SD) /110 & $40.50( \pm 14.04)$ & $20.69( \pm 9.80)$ \\
\hline $\begin{array}{l}\text { Peak Nasal Inspiratory Flow, } \\
\text { mean }( \pm S D) L / m i n\end{array}$ & $75.0( \pm 21.21)$ & $112.5( \pm 26.30)$ \\
\hline
\end{tabular}

(100\%). The influence of the chosen surgical technique accounts for $4.53 \%$ of the total variance, which was not significant. There was no significant effect of gender on the response to surgery (4.09\% of the variance).

\section{Clinical relevance of ENVC grading system}

We observed a significant improvement in the SNOT-22 score (Figure 4). Correlation between our ENVC score and the SNOT-22 score was moderate (Spearman $\mathrm{r}^{2}=0.48$ ), suggesting they are measuring related but different parameters. Our devised score examines the degree of clinical external valve collapse. The SNOT-22 score evaluates a cluster of interconnected symptoms including general health issues related to the nose. In our group of patients presenting ENVC, the responsiveness of our ENVC score was higher than the SNOT-22 score (Figure 4). Correlation between our ENVC score and the peak nasal inspiratory flow was moderate (Spearman $r^{2}=0.66$ ). Although the main concern of our patients was nose blockage, the high variability in the measurement of the peak nasal inspiratory flow resulted in this moderate correlation (results summarized in Table 3 ).

\section{Discussion}

We have devised and validated a subjective measurement score for ENVC ranging from 0 to 4. This outcome tool was able to reflect severity of collapse. It showed significant reliability and responsiveness to surgical treatment. ENVC is an unusual presentation and only a few studies have addressed it specifically. This is the first study to propose a scoring system of ENVC and to validate it in 26 patients.

In recent years, there has been an expansion in the number and use of instruments to measure the outcomes in functional septorhinoplasty surgery. Patient-derived questionnaires aim to quantify all aspects of a patient's experience. Clinical testing using the Cottle's manoeuvre or nasal strips ${ }^{(13)}$ evaluates valvular nasal obstruction and our ENVC score falls into this category of clinical evaluation. Objective measurements of nasal patency 
(peak nasal inspiratory flow, rhinomanometry, acoustic rhinometry) offer valuable pre and post operative evidence, however confounding factors need to be controlled ${ }^{(14,15)}$. Our score can be easily combined with other tools to refine the diagnosis, plan a surgical strategy and measure the outcome.

We appreciate the term ENVC is not universally adopted, however, it is used in this study for ease of anatomical localization. The alar region is dependent on the integrity of the lower lateral cartilages and their points of fixation. These points of fixation mirror the three major tip support mechanisms which include the integrity of lower lateral cartilage itself as well as the medial crura/footplate attachment to the septum and the scroll area attachment to the upper lateral cartilage ${ }^{(6,16)}$. ENVC occurs as a result of violation of all 3 major tip support mechanisms. Typically the weakened areas in the lower lateral cartilage include the intermediate and lateral crura. The commonest weakened area of fixation is the reduced scroll area attachment to the upper lateral cartilage. The fibrous attachment of the lateral crura to the upper lateral cartilage prevents the lateral crura from medialisation and subsequent collapse, particularly on inspiration. The causes of scroll area weakness can be congenital or acquired ${ }^{(6,16)}$. The commonest congenital cause is a cleft deformity and the commonest acquired cause is secondary to trauma or septorhinoplastic surgery. All our cases are pathological although the concept of physiological collapse needs to be considered.

There are limitations to our validation process in that we used standardized photographs to evaluate the reproducibility of our ENVC score ${ }^{(17)}$, however, each photograph was carefully taken so as to depict the important distinction between the scores. The take home message, however, is to score patients in the clinical setting and not by their clinical photograph as it may not capture the true extent of collapse. We could have scored a normal population without nasal blockage enabling us to have a control arm but owing to the study design this was not undertaken. The total score in reality combines the dynamic and static assessments and therefore does not differentiate the static blockage from the dynamic collapse. This is more apparent in congenital stenosis such as a cleft palate nasal deformity. However, in the cleft nose where there is a static ENV deformity, this scoring system can be applied by comparing the abnormal side with the normal side.

ENVC is an unusual presentation in primary septorhinoplasty surgery with an incidence of $10 \%$ in our revision septorhinoplasty practice ${ }^{(18)}$, unlike internal nasal valve collapse, which is well described in the literature and classically assessed through the Cottle's manoeuvre ${ }^{(13)}$. The clinical distinction between internal and external nasal valve dysfunction needs to be addressed in the clinical work-up. As objective measures of nasal patency cannot provide this distinction, some studies combine the two nasal valve dysfunctions despite the anatomical difference ${ }^{(19)}$. Gruber and colleagues described a classification of nasal valve dysfunction using nasal strips. This system is more accurate than the Cottle's manoeuvre and allows to distinguish between internal and external nasal valve collapse but not severity ${ }^{(8,13)}$. Our scoring system is complementary in providing assessment of ENVC and is user friendly in the out-patient setting. Physiological or paradoxical alar collapse occurs when one nostril is completely blocked as a result of, for example, a deviated nasal septum with resultant contralateral alar collapse on deep inspiration. This is resolved following correction of the unilateral blockage and needs careful assessment before consideration of alar reconstruction.

There is now an increasing need to quantify nasal blockage so as to determine efficacy of treatment. Objective measures of nasal patency (peak nasal inspiratory flow, rhinomanometry, acoustic rhinometry) have limitations in their use in the clinical setting with poor correlation with subjective patient improvement ${ }^{(15,20)}$. Quality of life questionnaires (SNOT-22 score, visual analogue scale) have been shown to accurately reflect nasal dysfunction and improve with septorhinoplasty surgery ${ }^{(21-23)}$. Quality of life questionnaires are particularly important in monitoring nasal valve surgery ${ }^{(11,24,25)}$.

The ENVC score is easy to implement in daily clinical practice. Our score evaluates severity of collapse on each side separately, which is pivotal in the surgical plan ${ }^{(11)}$ and we propose the ENVC score should be assessed and documented during clinical examination, as is the case, for internal nasal valve collapse.

\section{Conclusion}

We have devised and validated a subjective measurement score for ENVC ranging from 0 to 4. This grading system was reliable and responsive to functional septorhinoplasty.

\section{Acknowledgement}

No financial disclosure.

\section{Authorship contribution}

All authors were members of the RNTNE Rhinology \& Facial plastic team and contributed to the care of the patients. ALP and $P A$ designed the study, and together with $S A, I K, H C$ and $M B$ collected the data. ALP, PA, SA and IK were involved in literature search. PA devised the scoring system and conducted all surgical procedures. Data interpretation and writing were performed by ALP and PA.

\section{Conflict of interest}

None to declare. 


\section{References}

1. Spielmann PM, White PS, Hussain SS. Surgical techniques for the treatment of nasal valve collapse: a systematic review. Laryngoscope. 2009; 119: 1281-1290.

2. Rhee JS, Weaver EM, Park SS, et al. Clinical consensus statement: Diagnosis and management of nasal valve compromise. Otolaryngol Head Neck Surg. 2010; 143: 48-59.

3. Most SP. Trends in functional rhinoplasty. Arch Facial Plast Surg. 2008; 10: 410-413.

4. Murakami C. Nasal valve collapse. Ear, Nose, Throat J. 2004; 83: 163-164.

5. Schalek P, Hahn A. Anterior septal deviation and contralateral alar collapse. B-ENT. 2011; 7: 185-188.

6. Fischer H, Gubisch W. Nasal valves - importance and surgical procedures. Facial Plastic Surg. 2006; 22: 266-280.

7. Wittkopf M, Wittkopf J, Ries WR. The diagnosis and treatment of nasal valve collapse. Curr Opin Otolaryngol Head Neck Surg. 2008; 16: 10-13.

8. Gruber RP, Lin AY, Richards T. A predictive test and classification for valvular nasal obstruction using nasal strips. Plast Reconstr Surg. 2010; 126: 143-145.

9. Rhee JS, Arganbright JM, MCMullin BT, Hannley M. Evidence supporting functional rhinoplasty or nasal valve repair: A 25-year systematic review. Otolaryngol Head Neck Surg. 2008; 139: 10-20.

10. Lieberman DM, Most SP. Lateral nasal wall suspension using a bone-anchored suture technique. Arch Facial Plast Surg. 2010; 12: 113.

11. Lindsay RW. Disease-specific quality of life outcomes in functional rhinoplasty. Laryngoscope. 2012; 122: 1480-1488.

12. Khalil HS, Foxton CR, Mitchell-Innes AM. Septoplasty with alar battens for the treatment of alar collapse: how we do it. Clin Otolaryngol. 2011; 36: 575-578.

13. Gruber RP, Lin AY, Richards T. Nasal strips for evaluating and classifying valvular nasal obstruction. Aesthetic Plast Surg. 2011; 35: 211-215.

14. Nathan RA, Eccles R, Howarth PH, Steinsvag SK, Togias A. Objective monitoring of nasal patency and nasal physiology in rhinitis. Allergy Clin Immunol. 2005; 115: S442-5459.

15. Eccles R. A guide to practical aspects of measurement of human nasal airflow by rhinomanometry. Rhinology. 2011; 49: 2-10.

16. Timperley D, Stow N, Srubiski A, Harvey R, Marcells G. Functional outcomes of structured nasal tip refinement. Arch Facial Plast Surg. 2010; 12: 298-304.

17. Swamy RS, Most SP. Pre- and postoperative portrait photography: standardized photos for various procedures. Facial Plast Surg Clin North Am. 2010; 18: 245-252.

18. Boahene KD, Hilger PA. Alar rim grafting in rhinoplasty: indications, technique, and outcomes. Arch Facial Plast Surg. 2009; 11: 285-289.

19. Eduardo Nigro C, Faria Aguar Nigro J, Mion O, Ferreira Mello J, Jr., Louis Voegels R, Roithmann R. A systematic review to assess the anatomical correlates of the notches in acoustic rhinometry. Clin Otolaryngol 2009; 34: 431-437.

20. Boyce JM, Eccles R. Assessment of subjective scales for selection of patients for nasal septal surgery. Clin Otolaryngol 2006; 31:
297-302.

21. Hytonen ML, Lilja M, Makitie AA, Sintonen $H$, Roine RP. Does septoplasty enhance the quality of life in patients? Eur Arch Otorhinolaryngol. 2012; 269: 2497-2503.

22. Hopkins C, Gillett S, Slack R, Lund VJ, Browne JP. Psychometric validity of the 22-item Sinonasal Outcome Test. Clin Otolaryngol 2009; 34: 447-454.

23. Poirrier AL, Ahluwalia S, Goodson A, Ellis M, Bentley M, Andrews P. Is the Sino-Nasal Outcome Test-22 a suitable evaluation for septorhinoplasty? Laryngoscope 2012; 123 : 76-81.

24. Rhee JS, Poetker DM, Smith TL, Bustillo A, Burzynski M, Davis RE. Nasal valve surgery improves disease-specific quality of life. Laryngoscope 2005; 115: 437-440.

25. Most SP. Analysis of outcomes after functional rhinoplasty using a disease-specific quality-of-life instrument. Arch Facial Plast Surg. 2006; 8: 306-309.

Anne-Lise Poirrier

Sart-Tilman B35

ENT, Head and Neck Surgery

4000 Liege

Belgium

Tel: +32-4-366 7269

E-mail: annelise@poirrier.be 